\title{
Protein therapy using MafA fused to a polyarginine transduction domain attenuates glucose levels of streptozotocin-induced diabetic mice
}

\author{
JUN LU ${ }^{1 *}$, LINGJING LIN $^{1 *}$, HUIYUE DONG ${ }^{1 *}$, XIN MENG $^{1}$, FANG FANG ${ }^{2}$, \\ QINGHUA WANG ${ }^{1}$, LIANGHU HUANG ${ }^{1}$ and JIANMING TAN ${ }^{1}$
}

${ }^{1}$ Fujian Provincial Key Laboratory of Transplant Biology, Fuzhou General Hospital, Xiamen University, Fuzhou,
Fujian 350025; ${ }^{2}$ Department of Stomatology, Fujian Provincial Hospital, Fuzhou, Fujian 350001, P.R. China

Received February 25, 2016; Accepted February 21, 2017

DOI: $10.3892 / \mathrm{mmr} .2017 .6536$

\begin{abstract}
Ectopic expression of musculo aponeurotic fibrosarcoma BZIP transcription factor (Maf) A, has previously been demonstrated to induce insulin expression in non- $\beta$-cell lines. Protein transduction domains acting as an alternative delivery strategy may deliver heterogeneous proteins into cells. A sequence of 11 arginine residues (11R) has been demonstrated to act as a particularly efficient vector to introduce proteins into various cell types. The present study constructed 11R-fused MafA to achieve transduction of the protein into cellular membranes and subsequently examined the therapeutic effect of the MafA-11R protein in streptozotocin-induced diabetes. A small animal imaging system was used to demonstrate that $11 \mathrm{R}$ introduced proteins into cells. The MafA-11R protein was then injected into the tale vein of healthy male mice, and western blot analysis and immunofluorescence staining was performed to identify the location of the recombinant protein. Ameliorated hyperglycemia in the MafA-11R-treated diabetic mice was demonstrated via the improved intraperitoneal glucose tolerance test (IPGTT) and glucose-stimulated insulin release. Furthermore, insulin producing cells were detected in the jejunum of the MafA-11R treated mice. The results of the present study indicated that MafA-11R delivery may act as a novel and potential therapeutic strategy for the future and will not present adverse effects associated with viral vector-mediated gene therapies.
\end{abstract}

Correspondence to: Professor Jianming Tan or Dr Jun Lu, Fujian Provincial Key Laboratory of Transplant Biology, Fuzhou General Hospital, 156 Xierhuan Road, Fuzhou, Fujian 350025, P.R. China

E-mail: tanjm156@xmu.edu.cn

E-mail: junlu.heather@xmu.edu.cn

*Contributed equally

Key words: musculo aponeurotic fibrosarcoma BZIP transcription factor A, protein transduction domain, 11 arginine, diabetes

\section{Introduction}

Insulin gene expression is restricted to pancreatic $\beta$-cells in adult mammals. Transcription of the insulin gene is controlled by numerous factors, including pancreatic and duodenal homeobox (Pdx) 1, neuronal differentiation (NeuroD) and musculo aponeurotic fibrosarcoma BZIP transcription factor (Maf) A, which bind to 5'-cis-regulator elements in the enhancer region (1). MafA is a basic leucine zipper transcription factor, which controls expression of the insulin gene via a rat insulin promoter element (RIPE) 3b regulatory element (2). MafA deficient mice exhibit age-dependent glucose intolerance and abnormal islet architecture, suggesting that MafA is primarily involved in $\beta$-cell formation and functionality (3). Ectopic expression of MafA induces insulin production in various non- $\beta$-cell lines ( $\alpha$ TC6, AR42J and IEC- 6 ) (4), indicating that MafA exhibits the potential to induce insulin-producing cells. However, the majority of strategies involving the use of viruses as vectors for gene delivery result in safety concerns.

Protein transduction domains (PTDs) are small cationic peptides. Numerous PTD-fused full length functional proteins have been demonstrated to transduce cells and tissues $(5,6)$. This ability of the PTDs to transport intact proteins and allow them to penetrate the cell membrane, provides an alternative route of protein therapy in diseases. Of all the transduction peptides tested, tyrosine aminotransferase or eleven arginine residues $(11 \mathrm{R})$ demonstrate the greatest transduction efficiency $(7,8)$. Recombinant polyarginine-fused reprogramming factors have been used to facilitate proteins to generate induced pluripotent stem cells $(9,10)$.

It has been previously demonstrated that MafA, in conjuction with Pdx1 and NeuroD, may induce insulin gene expression in the liver, however this was not observed when MafA was administered alone (11). Conversely, adenovirus transduction with MafA alone promotes insulin production from intestinal cells (12), as the transcription factors Pdx1, neurogenin (Neurog3), NeuroD, NK 2 homeobox 2 and Paired box (Pax) 4, which are essential for pancreatic $\beta$-cell differentiation, are already expressed in the intestine $(13,14)$. The present study therefore delivered the MafA-11R recombinant protein to mice with streptozotocin-induced diabetes 
and monitored if the construct promoted the differentiation of intestinal cells into insulin producing cells. mCherry-11R served as a control protein, of which the distribution was observed via a small animal imaging system.

\section{Materials and methods}

Plasmid construction. The synthetic 11R cDNA sequence was cloned into the HindIII and XhoI sites of pET32a (EMD Millipore, Billerica, MA, USA) and subsequently termed pET32a(+)-11R. Full-length mouse MafA was amplified from mouse pancreas cDNA (cat. no. 9533; Takara Biotechnology Co., Ltd., Dalian, China) and mCherry was amplified from pmCherry-C1 (Clontech Laboratories, Inc., Mountainview, CA, USA) by polymerase chain reaction (PCR). The primer sequences were as follows: forward CGGGATCCATGG CCGCGGAGCTGGCGAT and reverse CCCAAGCTTCAG AAAGAAGTCGGGTGCGC for mouse mafA; forward CGGGATCCATGGTGA GCA AGGGCGAGGA and reverse CCCAAGCTTCTTGTACAGCTCGTCCATGC for mCherry. PrimeSTAR HS DNA polymerase (cat. no. R010A; Takara Biotechnology Co., Ltd.) was used for PCR with the following conditions: $95^{\circ} \mathrm{C}$ for $5 \mathrm{~min}$, followed by 35 cycles of denaturation at $95^{\circ} \mathrm{C}$ for $15 \mathrm{sec}$ and annealing and extension at $58^{\circ} \mathrm{C}$ for $1 \mathrm{~min}$. The PCR products were cloned into double-digested (BamHI and HindIII) pET32a(+)-11R vector. The resulting vectors were termed pET32a-MafA-11R and pET32a-mCherry-11R. In brief, the vectors were tagged with a 6-histidine ladder, followed by MafA or mCherry cDNA, then 11-R flanked by glycine and glutamic acid residues in the $\mathrm{COOH}$ terminal (Fig. 1A). The insertion efficiency of MafA, mCherry and 11R cDNA sequences into the pET32a vector, and potential sequence mutations that appeared during the process, were assessed via sequencing. The vector without $11 \mathrm{R}$ served as the negative control.

Purification and identification of recombinant proteins. The $11 \mathrm{R}$ fusion proteins were expressed and purified as previously described (7). In brief, the constructed plasmids were transformed into BL21(DE3) E. coli that were then cultured with fresh Lysogeny broth (LB) (Amp+) at $200 \mathrm{rpm}$ at $37^{\circ} \mathrm{C}$ until optical density 600 reached 1.0 , as measured by a UV/VIS spectrophotometer. Isopropyl- $\beta$-D-thiog alactopyranoside (Sigma-Aldrich; Merck KGaA, Darmstadt, Germany) was added to a final concentration of $0.5 \mathrm{mM}$, and the cells were then incubated for $12 \mathrm{~h}$ at $25^{\circ} \mathrm{C}$. Cells were sonicated, and the supernatants were recovered and applied to a column of Ni-NTA agarose (Qiagen, Inc., Valencia, CA, USA). The purified proteins were dissolved in buffer A $(50 \mathrm{mM} \mathrm{KH} 2 \mathrm{PO} 4-\mathrm{HCl}$, $300 \mathrm{mM} \mathrm{NaCl}$ ) and visualized by SDS-PAGE and Coomassie blue staining (Fig. 1B).

Protein transduction and cytotoxicity assays. The cytotoxicity of MafA-11R was evaluated via WST-1 assay kit (Roche Applied Science, Mannheim, Germany), according to the manufacturer's protocol, prior to commencing of animal experiments in vivo. IEC-6 rat intestinal epithelial cells and NIH3T3 mouse embryonic fibroblast cells were obtained from the American Type Culture Collection (Manassas, VA, USA) and cultured in Dulbecco's modified Eagle's medium (DMEM) containing $10 \%$ fetal calf serum, $100 \mathrm{U} / \mathrm{ml}$ penicillin, and $100 \mu \mathrm{g} / \mathrm{ml}$ streptomycin (all from Hyclone; GE Healthcare Life Sciences, Logan, UT, USA). Cells were incubated with $0.5 \mathrm{mmol} / 1 \mathrm{mCherry}-11 \mathrm{R}$ or MafA-11R at $37^{\circ} \mathrm{C}$ for $24 \mathrm{~h}$ followed by $10 \%$ of WST-1 solution for $30 \mathrm{~min}$. The product in the resulting colored solution was measured using a microplate reader at a wavelength of $450 \mathrm{~nm}$.

Animals. All animal studies were approved by the Ethics Committee for Animal Experimentation and the Animal Welfare at the Fuzhou General Hospital (Fuzhou, China). Male C57BL/6 mice (age, 8 weeks; weight, $\sim 23 \mathrm{~g}$; provided by and bred at the Shanghai Laboratory Animal Center, Shanghai, China) were randomly assigned to the following experiments.

Small animal imaging of $m$ Cherry-11R protein. The distribution of the mCherry-11R protein was examined, which consisted of a 6-histidine tag and a set of consecutive 11R. In brief, $0.1 \mathrm{mg}$ mCherry-11R protein was injected into the caudal vein of mice. Recombinant mCherry protein without $11 \mathrm{R}$ was used as the negative control $(0.1 \mathrm{mg})$. Animals were sacrificed $12 \mathrm{~h}$ following injection of protein. An IVIS 200 series system with a cooled charge-coupled-device camera (Caliper Life Sciences, Waltham, MA, USA) was utilized to image major organs. Acquisition times were from $1 \mathrm{sec}$ to $1 \mathrm{~min}$ with varying binning factors depending on the light emission. Regions of interest were created around specific anatomic sites and light emission was measured as photons $/ \mathrm{sec} / \mathrm{cm}^{2} / \mathrm{sr}$ (photon flux) using Living Image Software Version 4.3.1 (PerkinElmer, Inc., Waltham, MA, USA).

MafA-11R protein in vivo tissue distribution. Mice were injected intravenously with $0.1 \mathrm{mg}$ MafA-11R. Following $12 \mathrm{~h}$, heart, liver, muscle, intestine, kidney and pancreas tissues from MafA-11R-treated mice were harvested. Cell extracts were prepared by lysing the tissues with CytoBuster Protein Extraction Reagent (cat. no. 71009; Millipore; Merck KGaA). Total protein quantification was performed using a bicinchoninic acid assay kit (Pierce; Thermo Fisher Scientific, Inc., Waltham, MA, USA). Protein samples (50 $\mu \mathrm{g})$ were resolved on $10 \%$ SDS-PAGE gels and then transferred to polyvinylidine fluoride membranes (Immobilon-P PVDF Membrane; EMD Millipore). Membranes were blocked with $5 \%$ bovine serum albumin (BSA; Sangon Biotech Co., Ltd., Shanghai, China) in TBST (50 mmol/l Tris- $\mathrm{HCl}, 150 \mathrm{mmol} / \mathrm{l}$ $\mathrm{NaCl}$, and $0.1 \%$ Tween-20) at room temperature for $30 \mathrm{~min}$ and then incubated with primary antibody against polyclonal anti-6-histidine (1:2,000; cat. no. 000000011922416001; Sigma-Aldrich, Merck KGaA) at $4^{\circ} \mathrm{C}$ overnight. Following this, the membranes were incubated with anti-mouse horseradish peroxidase-conjugated secondary IgG antibodies (1:1000; cat. no. 7076S, Cell Signaling Technology) at room temperature for $1 \mathrm{~h}$ and developed using a SuperSignal West Pico chemiluminescent kit (Pierce; Thermo Fisher Scientific, Inc.).

Diabetic mouse model preparation and treatment with protein. $\mathrm{C} 57 \mathrm{BL} / 6$ male mice $(\mathrm{n}=30)$ received an intraperitoneal injection with streptozotocin (STZ; $50 \mathrm{mg} / \mathrm{kg}$; Sigma Aldrich; Merck KGaA) to induce diabetes for 5 consecutive days (15). Animals with fasting blood glucose levels of $16 \mathrm{mM}$ 
A

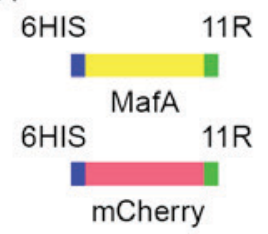

D

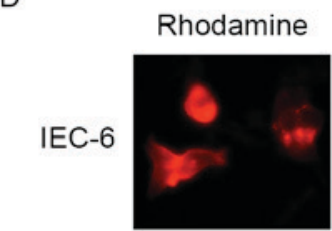

$\mathrm{NIH} 3 \mathrm{T3}$

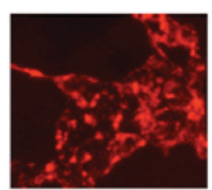

B 45

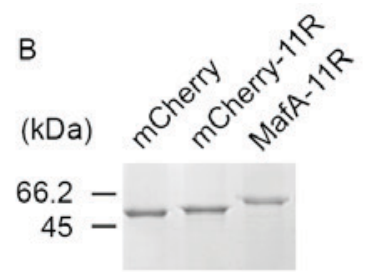

DAPI
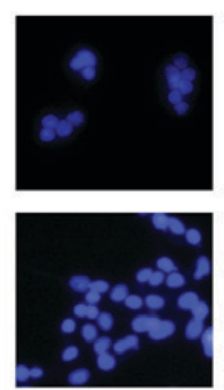

C
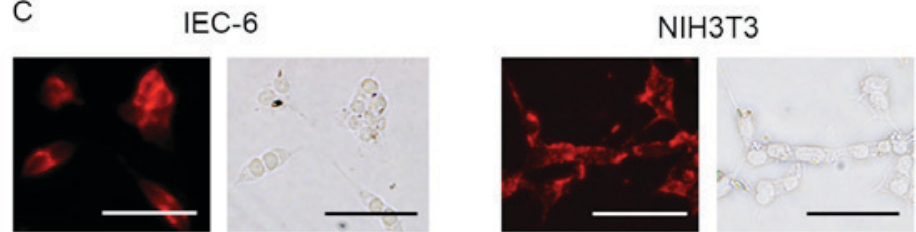

E

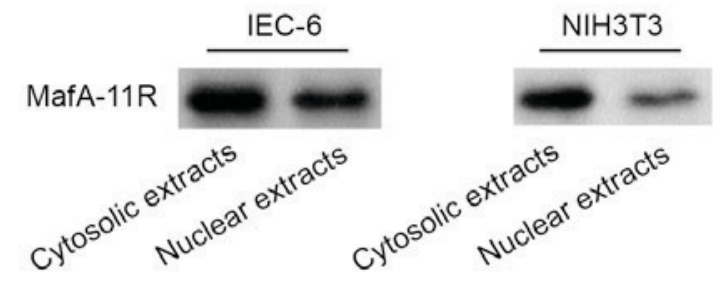

Figure 1. Transduction of 11R-fused proteins. (A) Structural diagram of mCherry-11R and MafA-11R. (B) SDS-PAGE visualization of the purified proteins. (C) Purified mCherry, mCherry-11R and MafA-11R proteins demonstrated via Coomassie blue staining. Each recombinant protein size is consistent with the predicted number of amino acids. (D) Transduction of mCherry-11R protein into IEC-6 and NIH3T3. IEC- 6 and NIH3T3 cells were treated with mCherry-11R protein for $4 \mathrm{~h}$, then rinsed well with PBS. (E) Western blot analysis using anti-6-histidine antibody on cytosolic and nuclear extracts of IEC-6 and NIH3T3 cells treated with MafA-11R protein. The results demonstrated are representative of three independent experiments. Bars, $100 \mu \mathrm{m}$. $6 \mathrm{HIS}, 6$-histidine ladder; MafA, musculo aponeurotic fibrosarcoma BZIP transcription factor A; 11R, 11 arginine; mCherry-11R, 11R-fused mCherry; MafA-11R, 11R-fused MafA protein.

for two consecutive readings $(\mathrm{n}=22)$ received protein treatment. The diabetic mice were injected with $0.1 \mathrm{mg}$ MafA-11R or $0.1 \mathrm{mg}$ mCherry-11R protein into the tail vein, 10 days post-STZ-injection. Following protein injection, non-fasting blood glucose levels were measured regularly.

Intraperitoneal glucose tolerance test. The normal, MafA-11R, and mCherry-11R-treated mice fasted overnight. They were administered glucose (1 mg/g body weight) intraperitoneally. Blood glucose levels were measured prior to and following glucose administration. Blood samples were obtained from the tail vein and analyzed using a glucometer.

Serum insulin measurements via enzyme-linked immunosorbent assay (ELISA). The normal, MafA-11R and mCherry-11R-treated mice fasted overnight and blood samples were collected at $15 \mathrm{~min}$ intervals following intraperitoneal glucose ( $1 \mathrm{mg} / \mathrm{g}$ body weight) administration. Serum insulin levels were determined via an ELISA kit (cat. no. EZRMI-13K; EMD Millipore).

Reverse transcription (RT) PCR. Total RNA from tissues was extracted using the TRIzol ${ }^{\circledR}$ reagent (Invitrogen; Thermo Fisher Scientific, Inc.) and cDNA was synthesized by reverse transcription using ReverTra Ace (Toyobo Co., Ltd., Osaka, Japan). The oligonucleotide primer sequences were as follows: Forward, GTCCCTCACCCTCCCAAA AG and reverse, GCTGCCTCAACACCTCAACCC for mouse actin and forward, CAGCAAGCAGGAAGCCTATC and reverse, GGAACCACAAAGGTGCTGCT for mouse insulin 2. The PCR was run for 35 cycles with 20 pmol primer under the following conditions: $94^{\circ} \mathrm{C}$ for $30 \mathrm{sec}, 56^{\circ} \mathrm{C}$ for $30 \mathrm{sec}$ and $72^{\circ} \mathrm{C}$ for $30 \mathrm{sec}$.
Quantitative RT-PCR (RT-qPCR). RT-qPCR was performed as previously described (16). The primer sequences used were as follows: Forward, CAACCGTGTAAATGCCACTG and reverse, TGCTACGGATGGACTGTTTG for mouse insulin 1; forward, AATGGTCGCCTCATTCTTTG and reverse, ATC AAGAGGGCTCCAGTCAA for mouse (glucose transporter) glut2; forward, CATCTCCCCATACGAAGTGC and reverse, ACGGGTCCTCTTGTTTTCCT for mouse pdx-1; forward GCTCCAGGGTTATGAGATCG and reverse, CTCTGCATT CATGGCTTCAA for mouse neuroD; forward, TCTACCAGC CAATCCCACAG and reverse, ATCATAACTCCGCCCATT CA for mouse pax6; forward, GGCTGCTGGTATCTTTGC TC, and reverse, CCAGAGCTTTGGCATTTAGC for mouse proprotein convertase (pc) $1 / 3$. The primers for $\beta$-actin and mouse insulin 2 were used as above. Relative fold changes in mRNA expression were calculated using the formula 2- $\Delta \Delta \mathrm{Cq}(17)$.

Immunofluorescence assay. The mice were sacrificed, and small intestine tissues were harvested, and fixed overnight with $4 \%$ paraformaldehyde. The fixed tissues were paraffin embedded and $5 \mu \mathrm{m}$ sections were cut and mounted onto slides. For insulin detection, the sections were rehydrated by bathing 3 times each in xylenes followed by isopropyl alcohol. Slides were then rinsed in distilled water. Antigen retrieval was performed with heated $10 \mathrm{mM}$ sodium citrate buffer, $\mathrm{pH}$ 6.0. A hydrophobic pen was used to outline the tissue. Tissue was blocked with PBS containing 5\% BSA (Sangon Biotech Co., Ltd.) and $0.05 \%$ Tween-20 for $30 \mathrm{~min}$ at room temperature. Mouse anti-6-histidine (1:500; cat. no. MA1-21315; Thermo Fisher Scientific, Inc.) or anti-insulin antibodies (1:250; cat. no. sc9168; Santa Cruz Biotechnology, Inc., Dallas, TX, USA) were diluted in blocking buffer and applied to the slides 

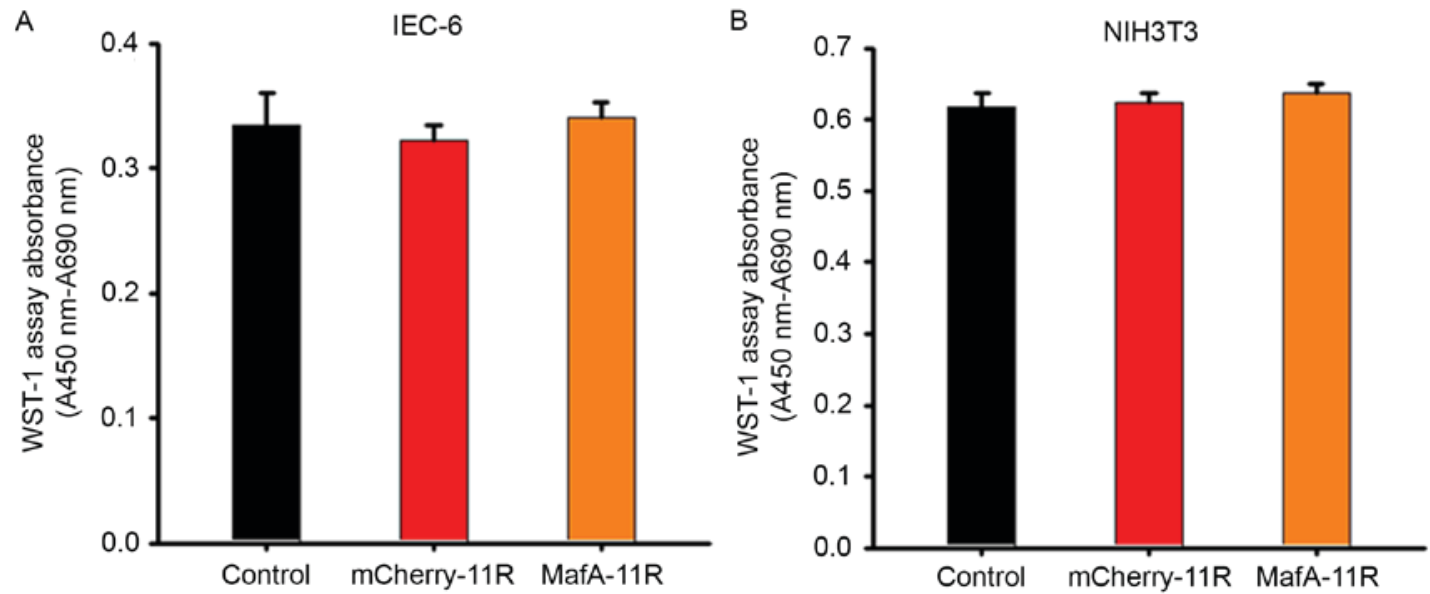

Figure 2. Cytotoxicity of the proteins detected via WST-1 assay. (A) IEC-6 or (B) NIH3T3 cells were incubated with mCherry-11R or MafA-11R. The graph demonstrates that mCherry-11R and MafA-11R did not significantly affect cell growth in vitro. Control, intact cells; MafA, musculo aponeurotic fibrosarcoma BZIP transcription factor A; 11R, 11 arginine; mCherry-11R, 11R-fused mCherry; MafA-11R, 11R-fused MafA protein.

A
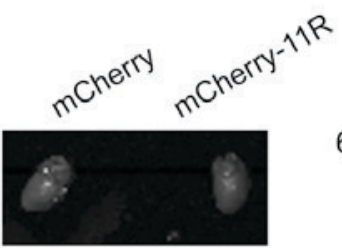

Liver

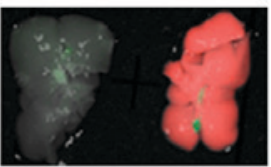

Muscle

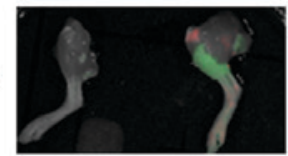

Intestine

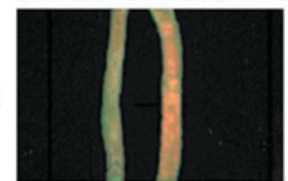

Kidney

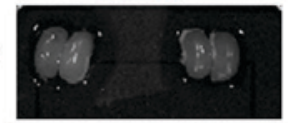

Pancreas

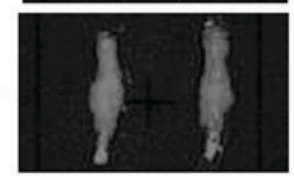

B

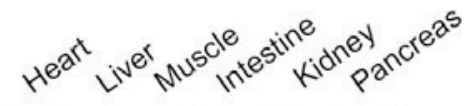

C

\section{$0 \mathrm{~h} 6 \mathrm{~h} 12 \mathrm{~h} 24 \mathrm{~h} 48 \mathrm{~h}$}

$6 \mathrm{His}$ (MafA-11R)

Actin
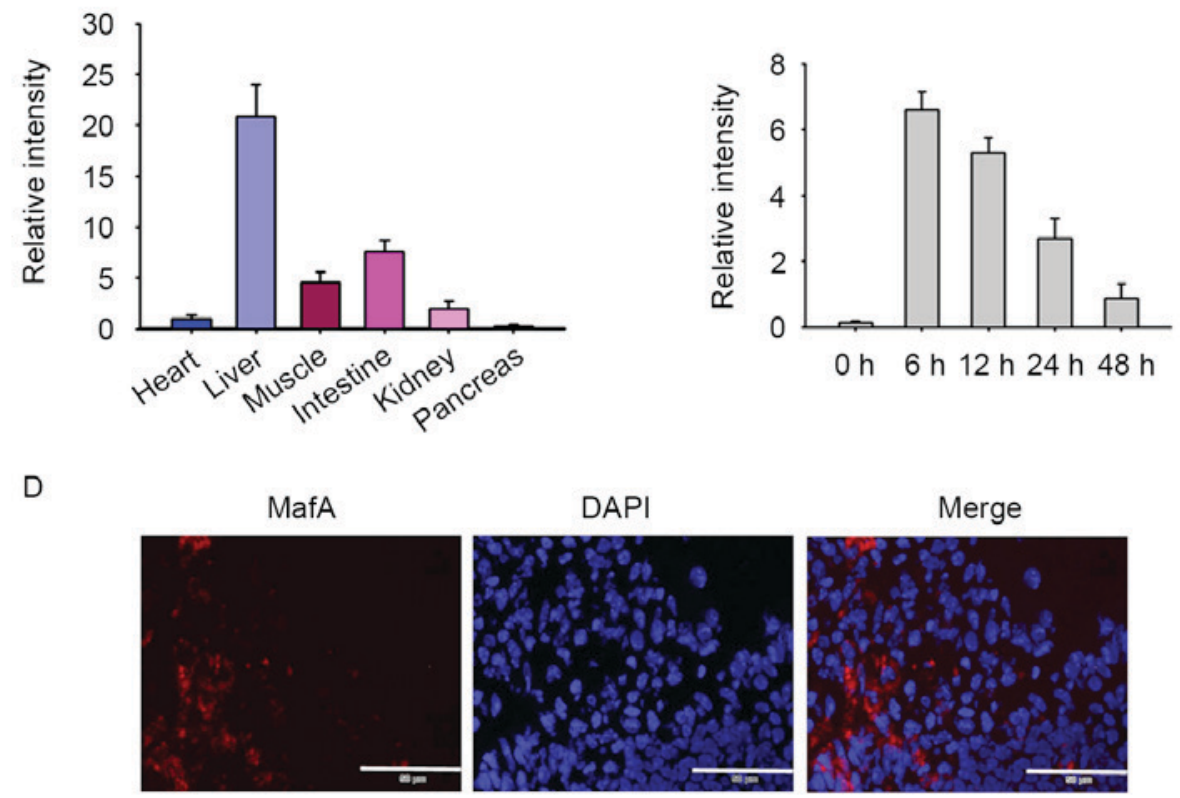
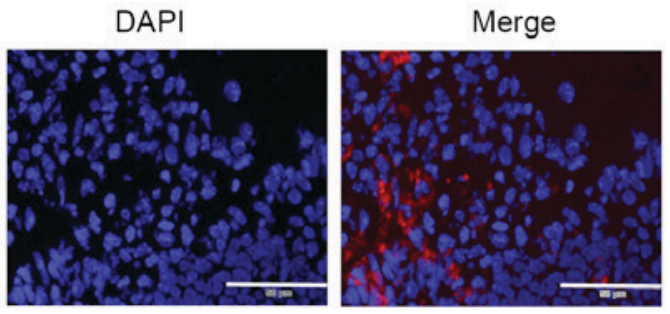

Figure 3. Localization of the 11R-fused proteins in vivo. (A) In vivo tissue distribution of mCherry-11R was observed by an IVIS 200 series system. Heart, liver, muscle, intestine, kidney and pancreas tissues were harvested $12 \mathrm{~h}$ following mCherry-11R intravenous injection. (B) The localization of MafA-11R was detected by western blotting. Major tissues were harvested $12 \mathrm{~h}$ following MafA-11R administration. Proteins were detected using anti-6-histidine or anti-actin antibody. The relative amount of MafA-11R protein was quantified by densitometry and the values normalized to actin. The heart reading was defined as 1 and the remaining values were divided by the heart reading. (C) Time course of penetration of MafA-11R proteins in small intestine. Normal C57BL/6 mice were injected intravenously with MafA-11R protein $(0.1 \mathrm{mg} / \mathrm{mouse})$. Intestinal samples were collected at the indicated times. The last reading was defined as 1 and the remaining values were divided by the last reading. (D) MafA-11R immunostaining of the intestine tissue. Intestine tissues were harvested $12 \mathrm{~h}$ following MafA-11R injection. Intestinal sections were immunostained with anti-6-histidine antibodies. Scale bars, $50 \mu \mathrm{m}$. 6 HIS, 6-histidine ladder; MafA, musculo aponeurotic fibrosarcoma BZIP transcription factor A; 11R, 11 arginine; mCherry-11R, 11R-fused mCherry; MafA-11R, 11R-fused MafA protein.

overnight at $4^{\circ} \mathrm{C}$. The sections were then incubated with rhodamine-conjugated goat anti-mouse or Dylight 594-conjugated donkey anti-rabbit $\operatorname{IgG}$ secondary antibodies (1:100; cat. nos. 115-025-075 and 715-585-150; Jackson ImmunoResearch
Laboratories, Inc., West Grove, PA, USA) and counterstained with DAPI (Sigma-Aldrich, Merck KGaA). Slides were analyzed using an epifluorescence microscope (X81; Olympus Corporation, Tokyo, Japan). 
Statistical analysis. Statistical analyses were performed using SPSS software version 17.0 (SPSS, Inc., Chicago, IL, USA). Data are presented as the mean \pm standard deviation. Data were analyzed using one- or two-way analysis of variance, followed by Bonferroni's multiple comparison test. $\mathrm{P}<0.05$ was considered to indicate a statistically significant difference.

\section{Results}

$11 R$ fusion proteins efficiently transduce cells in vitro. mCherry has previously been demonstrated to act as a useful monomeric red fluorescent protein, and therefore the mCherry-11R recombinant protein was used to visualize if $11 \mathrm{R}$ transduced fused proteins into the cells of interest. The IEC-6 and NIH3T3 cells were treated with mCherry-11R. Following $4 \mathrm{~h}$ incubation, a fluorescent signal was observed in the two cell lines ( $n=12$, each; Fig. 1C). To assess if the recombinant MafA-11R transduced into cells, IEC-6 and NIH3T3 were treated with His-tagged MafA-11R fusion protein. Immunostaining of His-tagged MafA protein revealed transduction of the MafA-11R protein ( $n=6$; Fig. 1D). The transduced MafA-11R protein was detected in the cytosolic and nuclear extracts of protein treated cells via western blot analysis, using anti-6-histidine antibody ( $\mathrm{n}=6$; Fig. 1E). These data demonstrated that $11 \mathrm{R}$ acted as a functional PTD and the recombinant proteins were capable of penetrating the cell membrane.

Cell viability assay. Prior to the commencement of the animal experiments in vivo, a cell viability assay was performed in vitro. The results demonstrated that the addition of mCherry-11R or MafA-11R fusion proteins did not inhibit the cell growth of the IEC-6 and NIH3T3 cell lines, which indicated that the proteins exhibited a low cytotoxicity $(n=48$ each; Fig. 2).

$11 R$ recombinant protein primarily present in liver and intestine cells in vivo. Firstly, as mCherry was easily observed by a small animal imaging system (17), the present study used it to detect and evaluate the distribution of 11R-fused recombinant proteins in vivo. mCherry-11R was constructed, however MafA-mCherry-11R was not used, as long recombinant proteins are susceptible to fracture. A total of $0.1 \mathrm{mg}$ mCherry-11R purified protein was injected into the tale vein of C57BL/6 mice and 11R-nonfused mCherry was applied as a negative control in this experiment ( $\mathrm{n}=6$ each). mCherry fluorescent signal is unable to be detected across the skin and muscle in protein-infused mice, therefore mice were sacrificed for detection of signal. It has previously been demonstrated that PTD alters the in vivo distribution of recombinant proteins by transducing them into cells and preventing rapid elimination into the urine. The present study examined recombinant protein distribution $12 \mathrm{~h}$ following injection. mCherry-11R was primarily present in the liver and intestine post-protein administration, however no fluorescent signal was detected by the small animal system in the 11R-nonfused mCherry control protein injected mice (Fig. 3A), which suggested that 11R facilitated the in vivo distribution of mCherry protein into the liver and intestine cells, and prevented its rapid elimination.
The MafA-11R protein may permeate into cells in vitro, however the in vivo tissue distribution of MafA-11R remains to be elucidated. To examine MafA-11R distribution, the C57BL/6 mice were injected with $0.1 \mathrm{mg}$ MafA-11R protein, major organs were harvested $12 \mathrm{~h}$ following injection and MafA-11R protein was then detected via anti-6-histidine immunoblotting. As indicated in the representative images of heart, liver, muscle, intestine, kidney and pancreas, the recombinant MafA-11R was concentrated primarily in the hepatocytes and intestine cells ( $n=6$; Fig. 3B). A low level of MafA-11R was additionally detected in the tissues of the kidney, muscle, heart and pancreas, however became undetectable at $24 \mathrm{~h}$ post injection (data not shown). Intestine samples were collected at various times and probed with anti-6-histidine antibody. The MafA-11R expression was evident in the intestine $6 \mathrm{~h}$ post injection and then gradually began to decline (Fig. 3C). Immunofluorescence examination confirmed the distribution of the MafA-11R protein in the intestine $12 \mathrm{~h}$ following injection (Fig. 3D).

MafA-11R protein decreases blood glucose levels in diabetic mice. To test the function of the MafA-11R protein in vivo, diabetic C57BL/6 mice received $0.1 \mathrm{mg}$ MafA-11R or nontherapeutic mCherry-11R protein (negative control; experimental design in schematic Fig. 1A). Non-fasting blood glucose levels were monitored as indicated. Diabetic mice that received MafA-11R injections 4 times achieved alleviation of hyperglycemia. Blood glucose levels were then kept at a low level and increased within 15 days of the first injection (Fig. 4B; $n=10-12$ ). A significant decrease was not detected in the blood glucose levels of the mCherry-11R injected control diabetic mice (Fig. 4B). The intraperitoneal glucose tolerance test (IPGTT; Fig. 4C) demonstrated that the MafA-11R injected mice exhibited a significantly improved IPGTT curve 15 days following the first injection ( $\mathrm{n}=5$, each), compared with the diabetic mice receiving mCherry-11R.

To further assess the ability of glucose-stimulated insulin release in the MafA-11R-treated diabetic mice, healthy normal and treated mice were challenged at day 15 post first injection with an intraperitoneal bolus of glucose. Sera collected from normal, MafA-11R-treated, or mCherry-11R-treated mice at 15 min post glucose injection were assayed for insulin. Serum insulin levels in MafA-11R-treated diabetic mice were 5.36 times greater at day 15 compared with those in the mCherry-11R-treated group (Fig. 4D), indicating an improvement in the ability of the MafA-11R-treated mice to react to a glucose challenge. Blood glucose levels were decreased at day 15 in MafA-11R-treated mice, however the insulin released $(3.27 \mu \mathrm{g} / \mathrm{l})$ following $15 \mathrm{~min}$ of glucose stimulation was decreased compared with that in normal nondiabetic mice $(9.74 \mu \mathrm{g} / \mathrm{l})$, suggesting functional immaturity of newly formed insulin producing cells ( $\mathrm{n}=6$ per group; $\mathrm{P}<0.05$ ).

$\beta$-cell regeneration in MafA-11R-treated mice was not observed (data not shown), therefore the present study hypothesized that MafA-11R promoted insulin expression in other tissues. Adenovirus-mediated overexpression of MafA may induce insulin gene expression in the rat small bowel, therefore the intestine was harvested at day 15 post injection in addition to the kidney, liver, heart and spleen. 


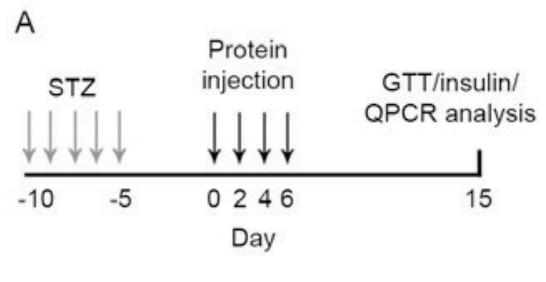

D

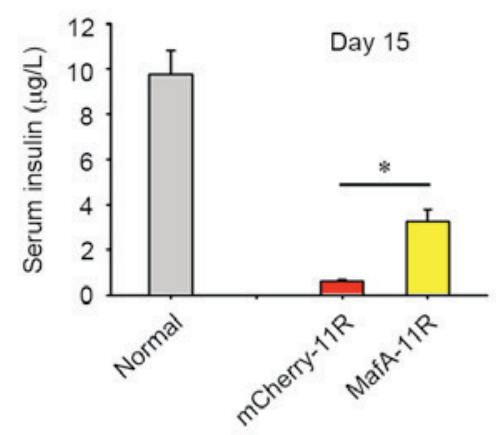

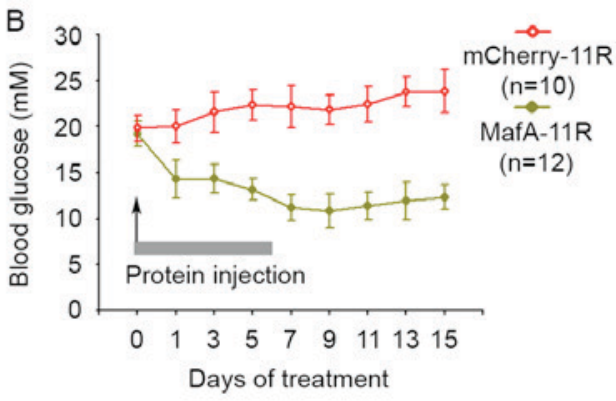

E

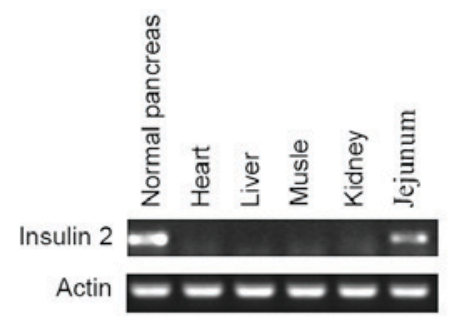

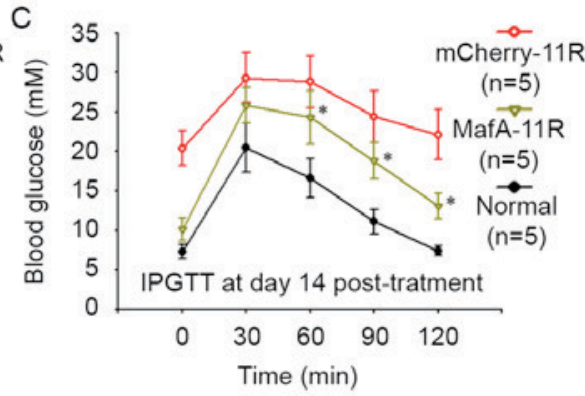

$\mathrm{F}$

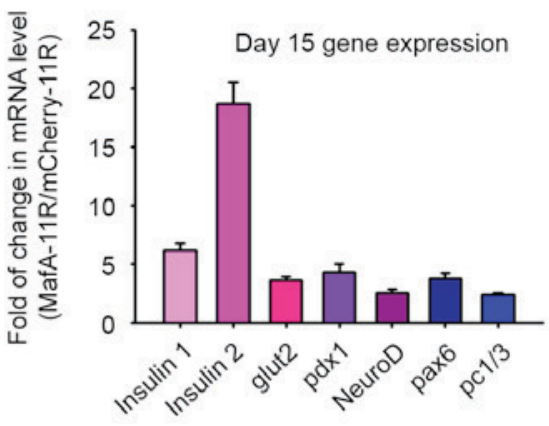

G

Normal pancreas
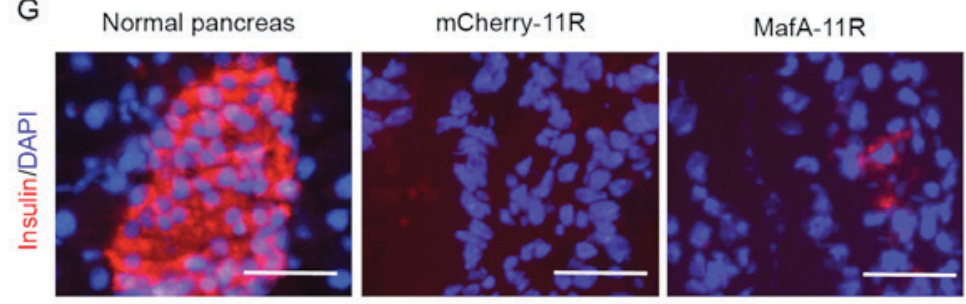

Figure 4. MafA-11R protein promotes the trans-differentiation of intestine cells into insulin-producing cells. (A) Experimental timeline, timing of STZ treatment with MafA-11R protein and the measurement of blood insulin levels, IPGTT and the determination of gene expression by RT-qPCR analyses. (B) Effects of MafA-11R protein on blood glucose levels. Diabetic C57BL/6 mice were treated with intravenous injections of $0.1 \mathrm{mg}$ MafA-11R or mCherry-11R 4 times (arrow) and blood glucose levels were determined by glucometer. (C) The IPGTT was performed as described in materials and methods, and blood glucose was measured at 0,30, 60,90 and $120 \mathrm{~min}$ in normal, MafA-11R or mCherry-11R-treated mice. ${ }^{*} \mathrm{P}<0.05$ compared with mCherry-11R. (D) Serum insulin levels were measured in MafA-11R- and mCherry-11R-treated mice 15 min following intraperitoneal glucose stimulation 15 days post-treatment. ${ }^{*}<0.05$ compared with mCherry-11R. (E) Expression of insulin 2 in organs. Total RNA from MafA-11R-treated diabetic mice at day 15 post-treatment and expression of insulin 2 were examined by RT-qPCR. Data are from $\geq 6$ mice/group and are representative of three independent experiments. (F) RT-qPCR analyses of pancreatic gene expression in intestines. Total RNA from diabetic mouse intestine (day 15 post-MafA-11R treatment) was analyzed via RT-qPCR for the expression of 7 key pancreatic genes (insulin 1, insulin 2, glut2, pdx1, neuroD, pax6 and pc1/3). (G) Insulin immunostaining. Paraffin sections from the intestine were stained with anti-insulin antibody. Bars, $50 \mu \mathrm{m}$. MafA, musculo aponeurotic fibrosarcoma BZIP transcription factor A; 11R, 11 arginine; MafA-11R, 11R-fused MafA protein; STZ, streptozotocin; IPGTT, intraperitoneal glucose tolerance test; RT-qPCR, reverse-transcription-quantitative polymerase chain reaction; mCherry-11R, 11R-fused mCherry; Pdx, pancreatic and duodenal homeobox 1; neuroD, neuronal differentiation; glut, glucose transporter 2; pc, proprotein convertase $1 / 3$; pax, paired box 6 .

The transcription of insulin 2 in MafA-11R-treated mice was detected via RT-PCR. Fig. 4E indicated the expression of insulin 2 in the jejunum, however not in other tissues. The control mCherry-11R-treated mice did not exhibit any signal in all the tissues tested.

To determine the molecular mechanism underlying MafA-11R-mediated insulin production in the intestine, RT-qPCR was used to examine the expression of key genes associated with pancreatic regeneration. MafA-11R treatment of diabetic mice at day 15 resulted in markedly upregulated levels of insulin 1 (6.17-fold), insulin 2 (18.64-fold), and slightly increased levels of glut2 (3.62-fold), pdx1 (4.29-fold), neuroD (2.53-fold), pax6 (3.75-fold) and pc1/3 (2.43-fold), compared with their corresponding control values (mCherry-11R treated intestine; Fig. 4F). An immunofluorescence study indicated that 15 days following MafA-11R treatment in diabetic mice, insulin-positive cells were present in the jejunum section (Fig. 4G). A total of 30 sections derived from 3 MafA-11R-treated mice were examined and the density of insulin-positive cells was $2.38 \pm 0.41 \mathrm{cell} / \mathrm{mm}^{2}$. Conversely, sections derived from mCherry-11R treated mice did not indicate insulin-positive cells. The amelioration of hyperglycemia and the detection of insulin-positive cells in the jejunum suggested that insulin was induced following MafA-11R administration.

\section{Discussion}

It has been previously demonstrated that PTD-fusion proteins are efficiently introduced into cultured cells and live tissues 
when injected into mice. PTDs may be applicable for various animal models that require repeated intracellular delivery of full length proteins in vivo $(18,19)$. The present study demonstrated that $11 \mathrm{R}$-fused proteins (mCherry-11R, MafA-11R) directly transduced the small intestine and the MafA-11R protein injection ameliorated hyperglycemia in mice with streptozotocin-induced diabetes. It was additionally observed that IPGTT and glucose-stimulated insulin release were improved in MafA-11R-treated diabetic mice and the MafA-11R protein induced insulin expression in the jejunum cells. The present study therefore constituted a demonstration that protein therapy in the form of in vivo MafA-11R delivery to animals may act as a novel therapeutic strategy, which does not present the adverse effects associated with viral vector-mediated gene therapies.

The results indicated that in vivo delivery of MafA-11R promoted the development of various jejunum cells into insulin producing cells. This effect was not observed in the liver or other tissues. It has previously been demonstrated that there is a developmental association between the gut and pancreas $(20,21)$. Various gut cells express the same molecules that have been demonstrated to convey glucose responsiveness in pancreatic $\beta$-cells. Commitment to the gut endocrine lineage requires the initiation of Neurog3 expression (22). Expression of NeuroD is required for the development of various gut endocrine cells (13). Pax4 and Pax6 are important in the differentiation into endocrine cells in the pancreas and intestine $(13,14)$. Therefore, the gut cells may be amenable to minimal engineering to secrete insulin, serving as $\beta$-cell surrogates. Various groups have demonstrated the successful induction of islet neogenesis in the liver using adenoviral vectors to deliver pancreatic transcription factors (23-25). MafA, in combination with Pdx-1 and NeuroD, resulted in the induction of long-term expression of insulin in the liver (26). In the present study, delivery of recombinant MafA did not induce insulin gene expression in the liver, which led to the hypothesis that combination with further transcription factors was necessary. Wang et al (27) demonstrated that a host response to an adenovirus, in combination with expression of pro-endocrine pancreatic transcription factors, is sufficient to induce insulin production in the liver of diabetic mice.

The present study demonstrated that MafA-11R effectively ameliorated hyperglycemia in diabetic mice, however there are potential obstacles to overcome prior to the use of this technique as a therapeutic strategy. One concern is the potential toxicity of MafA-11R, as the $11 \mathrm{R}$-fused protein exhibits potential to enter any tissue or cell type. The present study excluded the expression of insulin in tissues other than the intestine. The diabetic mice treated with MafA-11R appeared normal, without evidence of abnormal organ morphology. The animals gained body weight and exhibited reduced blood glucose levels. However, a full toxicity profile of MafA-11R is required in the future. Notably, the MafA-11R-induced insulin producing cells were still premature (the total amount of released insulin was decreased compared with that in normal mice), therefore repeated administration of MafA-11R, or administration in conjunction with transcription factors may be necessary and requires further investigation.

\section{Acknowledgements}

The present study was supported in part by The National Natural Science Foundation of China (grant nos. 81200560 and 81370948).

\section{References}

1. Docherty HM, Hay CW, Ferguson LA, Barrow J, Durward E and Docherty K: Relative contribution of PDX-1, MafA and E47/beta2 to the regulation of the human insulin promoter. Biochem J 389: 813-820, 2005.

2. Olbrot M, Rud J, Moss LG and Sharma A: Identification of beta-cell-specific insulin gene transcription factor RIPE3b1 as mammalian MafA. Proc Natl Acad Sci USA 99: 6737-6742, 2002.

3. Zhang C, Moriguchi T, Kajihara M, Esaki R, Harada A, Shimohata H, Oishi H, Hamada M, Morito N, Hasegawa K, et al: MafA is a key regulator of glucose-stimulated insulin secretion. Mol Cell Biol 25: 4969-4976, 2005.

4. Matsuoka TA, Kaneto H, Stein R, Miyatsuka T, Kawamori D, Henderson E, Kojima I, Matsuhisa M, Hori M and Yamasaki Y: MafA regulates expression of genes important to islet beta-cell function. Mol Endocrinol 21: 2764-2774, 2007.

5. Schwarze SR and Dowdy SF: In vivo protein transduction: Intracellular delivery of biologically active proteins, compounds and DNA. Trends Pharmacol Sci 21: 45-48, 2000.

6. Ho A, Schwarze SR, Mermelstein SJ, Waksman G and Dowdy SF: Synthetic protein transduction domains: Enhanced transduction potential in vitro and in vivo. Cancer Res 61: 474-477, 2001.

7. Matsushita M, Tomizawa K, Moriwaki A, Li ST, Terada H and Matsui H: A high-efficiency protein transduction system demonstrating the role of PKA in long-lasting long-term potentiation. J Neurosci 21: 6000-6007, 2001.

8. Matsui H, Tomizawa K, Lu YF and Matsushita M: Protein, Therapy: In vivo protein transduction by polyarginine (11R) PTD and subcellular targeting delivery. Curr Protein Pept Sci 4: 151-157, 2003.

9. Kim D, Kim CH, Moon JI, Chung YG, Chang MY, Han BS, Ko S, Yang E, Cha KY, Lanza R and Kim KS: Generation of human induced pluripotent stem cells by direct delivery of reprogramming proteins. Cell Stem Cell 4: 472-476, 2009.

10. Zhang H, Ma Y, Gu J, Liao B, Li J, Wong J and Jin Y: Reprogramming of somatic cells via TAT-mediated protein transduction of recombinant factors. Biomaterials 33: 5047-5055, 2012.

11. Kaneto H, Matsuoka TA, Nakatani Y, Miyatsuka T, Matsuhisa M, Hori M and Yamasaki Y: A crucial role of MafA as a novel therapeutic target for diabetes. J Biol Chem 280: 15047-15052, 2005.

12. Nomura S, Nakamura T, Hashimoto T, Nishio Y, Maegawa $H$, Kudo $\mathrm{M}$ and Kashiwagi A: MafA differentiates rat intestinal cells into insulin-producing cells. Biochem Biophys Res Commun 349: 136-143, 2006.

13. Schonhoff SE, Giel-Moloney M and Leiter AB: Minireview: Development and differentiation of gut endocrine cells. Endocrinology 145: 2639-2644, 2004.

14. van der Flier LG and Clevers H: Stem cells, self-renewal, and differentiation in the intestinal epithelium. Annu Rev Physiol 71: 241-260, 2009.

15. Wu KK and Huan Y: Streptozotocin-induced diabetic models in mice and rats. Curr Protoc Pharmacol Chapter 5: Unit 5.47, 2008.

16. Lu J, Li G, Lan MS, Zhang S, Fan W, Wang H and Lu D: Pax4 paired domain mediates direct protein transduction into mammalian cells. Endocrinology 148: 5558-5565, 2007.

17. Livak KJ and Schmittgen TD: Analysis of relative gene expression data using real-time quantitative PCR and the 2(-Delta Delta C(T)) Method. Methods 25: 402-408, 2001.

18. Zacharakis G, Kambara H, Shih H, Ripoll J, Grimm J, Saeki Y, Weissleder R and Ntziachristos V: Volumetric tomography of fluorescent proteins through small animals in vivo. Proc Natl Acad Sci USA 102: 18252-18257, 2005.

19. Toro A and Grunebaum E: TAT-mediated intracellular delivery of purine nucleoside phosphorylase corrects its deficiency in mice. J Clin Invest 116: 2717-2726, 2006.

20. Yamada S, Kanno H and Kawahara N: Trans-membrane peptide therapy for malignant glioma by use of a peptide derived from the MDM2 binding site of p53. J Neurooncol 109: 7-14, 2012. 
21. Slack JM: Developmental biology of the pancreas. Development 121 1569-1580, 1995.

22. Grapin-Botton A, Majithia AR and Melton DA: Key events of pancreas formation are triggered in gut endoderm by ectopic expression of pancreatic regulatory genes. Genes Dev 15: 444-454, 2001

23. Jenny M, Uhl C, Roche C, Duluc I, Guillermin V, Guillemot F, Jensen J, Kedinger $\mathrm{M}$ and Gradwohl G: Neurogenin3 is differentially required for endocrine cell fate specification in the intestinal and gastric epithelium. EMBO J 21: 6338-6347, 2002.

24. Kojima H, Fujimiya M, Matsumura K, Younan P, Imaeda H, Maeda M and Chan L: NeuroD-betacellulin gene therapy induces islet neogenesis in the liver and reverses diabetes in mice. Nat Med 9: 596-603, 2003.
25. Taniguchi H, Yamato E, Tashiro F, Ikegami H, Ogihara T and Miyazaki J: beta-cell neogenesis induced by adenovirus-mediated gene delivery of transcription factor pdx-1 into mouse pancreas. Gene Ther 10: 15-23, 2003.

26. Kaneto H, Matsuoka TA, Katakami $N$ and Matsuhisa $M$ : Combination of MafA, PDX-1 and NeuroD is a useful tool to efficiently induce insulin-producing surrogate beta-cells. Curr Med Chem 16: 3144-3151, 2009.

27. Wang AY, Ehrhardt A, Xu H and Kay MA: Adenovirus transduction is required for the correction of diabetes using Pdx-1 or Neurogenin-3 in the liver. Mol Ther 15: 255-263, 2007. 